\title{
Disseminated Spinal Epidural Abscess in an Immunocompetent Individual: A Case Report and Review of the Literature
}

\author{
Daisuke Usuda ${ }^{\mathrm{a}}$, , e, Yasuhiko Takic ${ }^{\mathrm{c}}$, Toshihide Izumida ${ }^{\mathrm{a}}$, Ryusho Sangen ${ }^{\mathrm{a}}$, \\ Toshihiro Higashikawad ${ }^{\mathrm{d}}$, Eiju Hatano ${ }^{\mathrm{c}}$, Mitsuteru Yokoyama ${ }^{\mathrm{c}}$, \\ Yuji Kasamaki ${ }^{\mathrm{a}}$
}

\begin{abstract}
Spinal epidural abscess (SEA) is an uncommon pyogenic infection, localized between the dura mater and vertebral periosteum, leading to significant morbidity and mortality. SEA development is connected with medical comorbidities and risk factors facilitating bacterial dissemination; multiple factors are believed to play a role, including aging, increased alcohol abuse, use of intravenous drugs, a greater prevalence of medical comorbidities, and increased rates of spinal surgery that furthers iatrogenic spinal infection. Here, we have reported the first known case of disseminated SEA in an immunocompetent individual. A 33-year-old Japanese woman visited our hospital due to 1 week of continuous fever, low back pain, and numbness of the entire left lower limb. She was diagnosed with disseminated SEA by complete spine magnetic resonance imaging scan, of unknown origin. She was treated for 13 days with piperacillin-tazobactam, then for 16 days with levofloxacin tablets; ultimately, she recovered without treatment complications. This case highlights the complicated pathology, diagnosis, and treatment of SEA. In addition, this case suggests the need for a careful and detailed examination when encountering patients presenting with fever, low back pain even in an immunocompetent individual; we should thoroughly investigate, including further image investigations, bacteriological and pathologic examination.
\end{abstract}

Keywords: Epidural abscess; Immunocompetence; Fever; Low back pain; Magnetic resonance imaging

Manuscript submitted October 5, 2020, accepted October 12, 2020

Published online October 21, 2020

aDepartment of General Medicine, Kanazawa Medical University Himi Municipal Hospital, 1130 Kurakawa, Himi-shi, Toyama-ken 935-8531, Japan

bDepartment of Infectious Diseases, Kanazawa Medical University, 1-1 Daigaku, Uchinada-machi, Ishikawa-ken 920-0293, Japan

'Department of Orthopedics, Kanazawa Medical University Himi Municipal Hospital, 1130 Kurakawa, Himi-shi, Toyama-ken 935-8531, Japan

${ }^{\mathrm{d}}$ Department of Geriatric Medicine, Kanazawa Medical University Himi Municipal Hospital, 1130 Kurakawa, Himi-shi, Toyama-ken 935-8531, Japan ${ }^{e}$ Corresponding Author: Daisuke Usuda, Department of General Medicine, Kanazawa Medical University Himi Municipal Hospital, 1130 Kurakawa, Himi-shi, Toyama-ken 935-8531, Japan. Email: united19771108@yahoo.co.jp

doi: https://doi.org/10.14740/jmc3603

\section{Introduction}

Spinal epidural abscess (SEA) is an uncommon pyogenic infection, localized between the dura mater and vertebral periosteum, leading to significant morbidity and mortality [1-10]. SEA development is connected with medical comorbidities and risk factors facilitating bacterial dissemination [9]. Multiple factors are believed to play a role, including aging, increased alcohol abuse, use of intravenous (IV) drugs, a greater prevalence of medical comorbidities (e.g., diabetes mellitus (DM) or the human immunodeficiency virus (HIV)), and increased rates of spinal surgery that furthers iatrogenic spinal infection [3].

SEA exists in both acute and chronic presentations [1]. Acute SEA generally has a duration of no more than 2 weeks, accompanied by fever and signs of systemic inflammation from a hematogenous source [1]. Compare this with subtle, afebrile, and long-standing chronic SEA, which is a result of a direct extension of the vertebral osteomyelitis (VO) [1]. Acute forms generally exist posterior to the spinal cord, while chronic forms are often found anterior to the spinal cord [1]. In acute cases, gross pathology will generally be purulent and exudative, whereas chronic cases will have granulation tissue [1]. SEA can also be broadly distinguished into two categories: primary SEA, caused by pathogen hematogenous dissemination via contiguous infected tissue, hematogenous spread, or iatrogenic inoculation; or secondary SEA, which is the result of direct pathogen inoculation $[5,7,9]$.

As far as we are aware, we have reported the first known case of disseminated SEA with unknown origin in an immunocompetent individual, together with a brief review of literature.

\section{Case Report}

A 33-year-old Japanese woman visited our hospital due to 1 week of continuous low-grade fever, low back pain, and numbness of the entire left lower limb. These symptoms occurred when she lifted a heavy suitcase. She had visited an orthopedic doctor and received a caudal block 4 days prior, followed by a high-grade fever and deteriorating low back pain starting 1 day prior. Her medical history included surgery to enlarge both breasts when she was 14 years old. She was not taking any 
medicines on a regular basis. She had a smoking history of five cigarettes a day starting at the age of 20 , and no history of drinking alcohol. She underwent periodic medical exams, and the latest one, which had been 2 months before, appeared normal. The patient was employed at a film factory, as a product inspector, and had no food or drug allergies. She had no history of casual sex, intramuscular injections, or IV drug use.

She was $153 \mathrm{~cm}$ tall, and weighed $42 \mathrm{~kg}$. Her vital signs were abnormal, with blood pressure of 104/85 $\mathrm{mm} \mathrm{Hg}$, a heart rate of 123 regular beats $/ \mathrm{min}$, a body temperature of $38.1^{\circ} \mathrm{C}$, oxygen saturation of $98 \%$ in ambient air, and a respiratory rate of 16/min; her Glasgow Coma Scale score was 15 (E4V5M6) points. She complained of percussion tenderness to the lower thoracic vertebra and the upper sacral bone. Her manual muscle testing score for the left lower limb was 4; on the other hand, nothing else abnormal, including skin or neurologic findings, was detected upon physical examination.

A routine laboratory examination of the patient revealed increased values of white blood cells, creatinine, C-reactive protein (CRP), and erythrocyte sedimentation rate (ESR), and decreased values of blood urea nitrogen and creatine kinase. On the other hand, other values were normal, including complete blood count ( $\mathrm{CBC})$, biochemistry, urine tests, and cerebrospinal fluid examination (Table 1). She also tested negative for rheumatoid factor, autoantibodies such as anti-nuclear antibodies, anti-neutrophil cytoplasmic antibodies, serologic test for hepatitis B and C, syphilis, HIV, thyroid hormone, soluble interleukin-2 receptor, flu test, and 2/2 sets of blood culture. Head and chest-abdomen computed tomography (CT) scans and transesophageal echocardiography revealed normal results. On the other hand, a complete spine magnetic resonance imaging (MRI) scan revealed a 0.5 - mm-thick layer of spinal epidural fluid in the posterior epidural space in the whole thoracic and anterior epidural space from the L5 to S2 vertebral body level region, which compressed the epidural space (Figs. 1a, b, and $2 \mathrm{a}$, respectively). These findings were revealed as low intensity in a T1-weighted image, and high intensity in a T2-weighted image. Other findings, such as discitis, were not confirmed.

At this point, we suspected a diagnosis of disseminated SEA, but the origin was still unknown. However, an orthopedic surgeon recommended against performing CT-guided percutaneous needle aspiration of the abscess, based on the locations of the lesions. The patient was hospitalized, and we started 13.5 $\mathrm{mg}$ /day of piperacillin-tazobactam, following which she gradually became symptom-free. However, on the 14th hospital day, she strongly requested that she be discharged from the hospital, so we changed her antibiotics to $500 \mathrm{mg} /$ day of levofloxacin tablets, and continued treatment. During her return visit, 16 days after her discharge, she was well. We confirmed normal values of inflammation biomarkers, including WBC, CRP, and ESR, and in addition we also confirmed abscess improvements in both the thoracic and sacral regions through an MRI scan (Figs. 1c, d, and 2c, respectively). Based on these findings, we ended her treatment that day without treatment complications.

\section{Discussion}

We present the first known case of disseminated SEA in an immunocompetent individual. Regarding etiology, she had no associated antecedent infections, including discitis, medical comorbidities, or risk factors that would facilitate SEA. In addition, we cannot consider the caudal block orthopedic doctor for etiology, because she already had symptoms when she visited the orthopedic doctor. The etiology in this case still remains unknown; however, there is a possibility of SEA progress due to primary hematogenous spread from another region, rather than ascending infection, considering the abscess location. Given this point of view, this case is extremely rare with regards to etiology. On the other hand, there may be a possibility that caudal block led to low back pain deterioration.

SEA is estimated to have an annual incidence of 2.4 cases per 100,000 individuals, with incidence of infection increasing with age $[11,12]$. SEA is rarely seen in individuals under the age of 20 ( 0.3 per 100,000 persons), with incidence levels over 20 times greater in older patients $(6.5$ per 100,000 persons over 70 years old) [11]. A prevalence peak can be seen from the fifth to seventh decades of life, and it is predominantly seen in males [9]. However, SEA incidence has shown an upward trend: the historical incidence is 0.2 - 2 cases per 10,000 hospital admissions, while more recent estimates have been 2 12.5 cases per 10,000 hospital admissions [3]. This increasing incidence of SEA has been attributed to greater prevalence of factors that may lead to predisposition, such as spinal procedures and instrumentation [5]. In this case, the patient was of atypical age for the occurrence of SEA.

There are no known classic history or physical exam findings that can be used to identify SEA easily and reliably [11]. The primary symptom, back pain, is a fairly common complaint, which is often associated with a number of other, less immediately serious conditions [11]. Mid-thoracic and lower thoracic spinal infectious processes, for example, can present as lower back pain [11]. In addition, patients with SEA may present with fever and neurologic deficits, and symptoms can also vary depending on the SEA location (for instance, cases with cervical involvement may present with dysphagia, while cases with thoracic involvement can potentially manifest with autonomic dysregulation $[2,11,13]$. Presenting with both back and radicular pain is more frequently seen with the acute form, and much less so for the chronic form [1]. Emergency providers are conventionally taught the "classic triad" of spinal pain, fever, and neurological deficit assessments, but this constellation of findings only appears in some $10-15 \%$ of cases $[3,7]$. Moreover, back pain and fever, the common early symptoms, are relatively nonspecific, and patients will generally seek medical attention at varying stages of disease progression [7, 14]. Additionally, in the event that the abscess is sufficiently large, patients can rapidly develop the neurologic signs of spinal injury, which may necessitate urgent neurosurgical intervention [15]. Furthermore, it can be particularly difficult to discover late neurological symptoms such as these in patients who are bedridden or chronically ill [7].

Prompt evaluation and treatment is necessary for any clinical concern for this diagnosis $[2,4,5,14]$. However, it can be difficult to diagnose SEA without significant clinical suspicion [2]. In addition, if it is not recognized, diagnosed, and treated in a timely manner, it can ultimately lead to patient outcomes such as permanent paralysis or deformity of the spine [11]. Un- 
Table 1. Routine Laboratory Examination on Admission

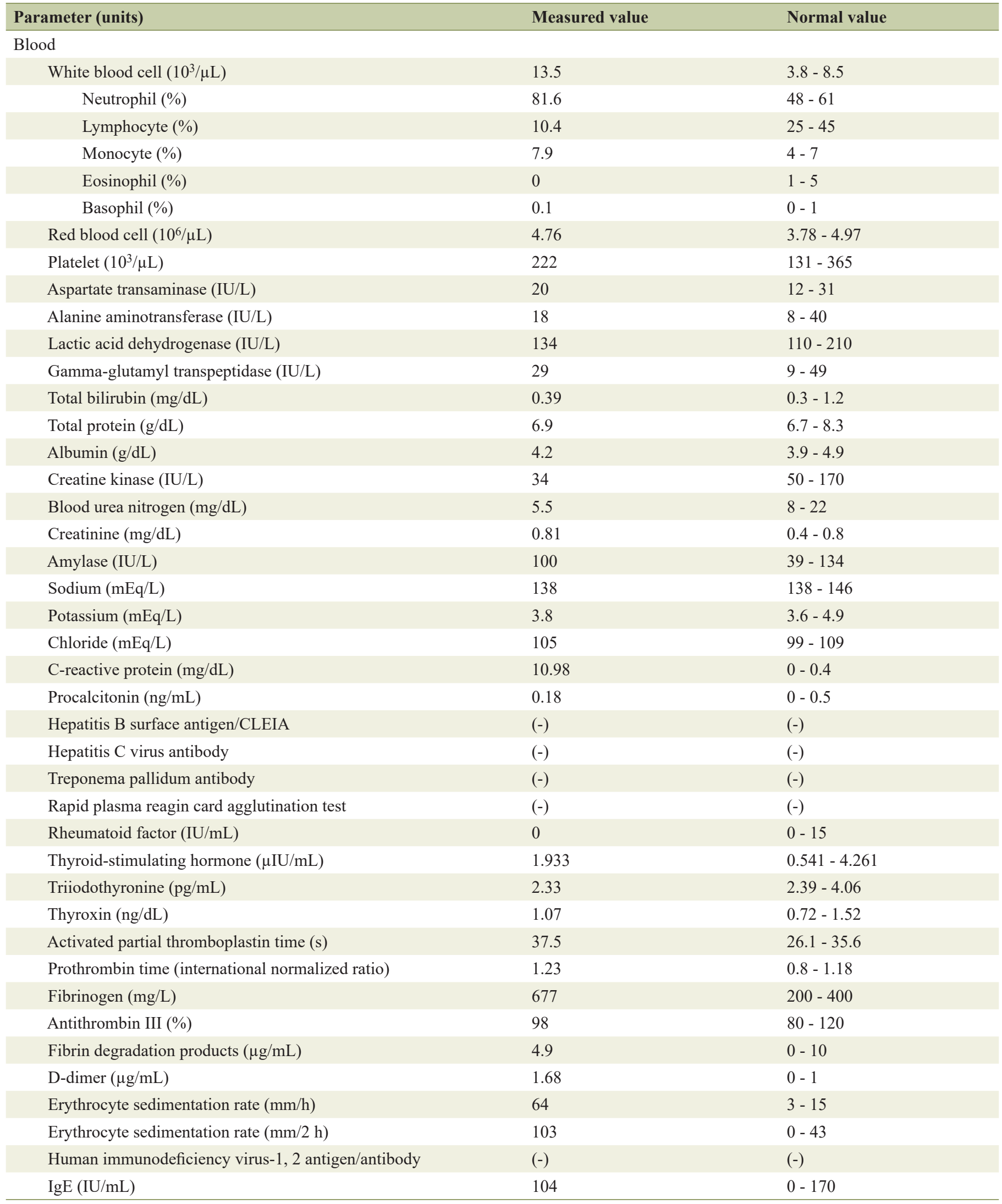


Table 1. Routine Laboratory Examination on Admission - (continued)

\begin{tabular}{|c|c|c|}
\hline Parameter (units) & Measured value & Normal value \\
\hline PR3-antineutrophil cytoplasmic antibody & $(-)$ & $(-)$ \\
\hline MPO-antineutrophil cytoplasmic antibody & $(-)$ & $(-)$ \\
\hline Antinuclear antibody (times) & $<40$ & $<40$ \\
\hline Anti-cardiolipin IgG (U/mL) & 3 & $0-10$ \\
\hline Urobilinogen & $(-)$ & $(-)$ \\
\hline Occult blood reaction & $(-)$ & $(-)$ \\
\hline Protein & $(-)$ & $(-)$ \\
\hline Sugar & $(-)$ & $(-)$ \\
\hline Nitrite & $(-)$ & $(-)$ \\
\hline White blood cells & $(-)$ & $(-)$ \\
\hline Streptococcus pneumoniae & $(-)$ & $(-)$ \\
\hline Legionella pneumoniae & $(-)$ & $(-)$ \\
\hline Pregnant reaction & $(-)$ & \\
\hline Flu A & $(-)$ & $(-)$ \\
\hline Flu B & $(-)$ & $(-)$ \\
\hline Mycoplasma pneumoniae antigen & $(-)$ & $(-)$ \\
\hline \multicolumn{3}{|l|}{ Cerebrospinal fluid } \\
\hline Protein $(\mathrm{mg} / \mathrm{dL})$ & 34.1 & \\
\hline Sugar (mg/dL) & 57 & \\
\hline Potassium (mEq/L) & 2.6 & \\
\hline Chloride $(\mathrm{mEq} / \mathrm{L})$ & 122.9 & \\
\hline
\end{tabular}

CLEIA: chemiluminescent enzyme immunoassay; PR3: proteinase 3; MPO: myeloperoxidase; Ig: immunoglobulin.

fortunately, diagnostic delays are not uncommon, with reports of a range of 11 - 59 days from symptom onset to diagnosis [11]. Therefore, it is necessary to maintain a high degree of clinical suspicion in order to rapidly diagnose and initiate treatment $[6,16]$.

First, if a patient is suspected of having SEA, a detailed neurologic exam is necessary [11]. Unfortunately, there are no pathognomonic signs or symptoms that can be used to confirm an SEA diagnosis; likewise, normal exam results do not exclude this diagnosis [11]. The "classic triad" SEA symptoms are insensitive markers; one case series found sensitivity values of $7.9 \%$, specificity values of $99 \%$, positive predictive val- ues of $83 \%$, and negative predictive values of $68 \%$ [11].

Unfortunately, objective neurologic findings have proven to be the exception rather than the rule: when present, they vary widely, from mild (radicular pain corresponding to the nerve root) to moderate (motor weakness, bowel or bladder dysfunction, or sensory loss) to severe (paralysis) [11]. When an abnormal neurologic exam is present, point tenderness should be cause for an urgent investigation into possible SEA, because delayed diagnosis can lead to permanent neurologic deficits [11]. Severity progression suggests an infectious spread into the epidural space, with compression of the nerve root and eventually the spinal cord [11]. Furthermore, a missed SEA di- 


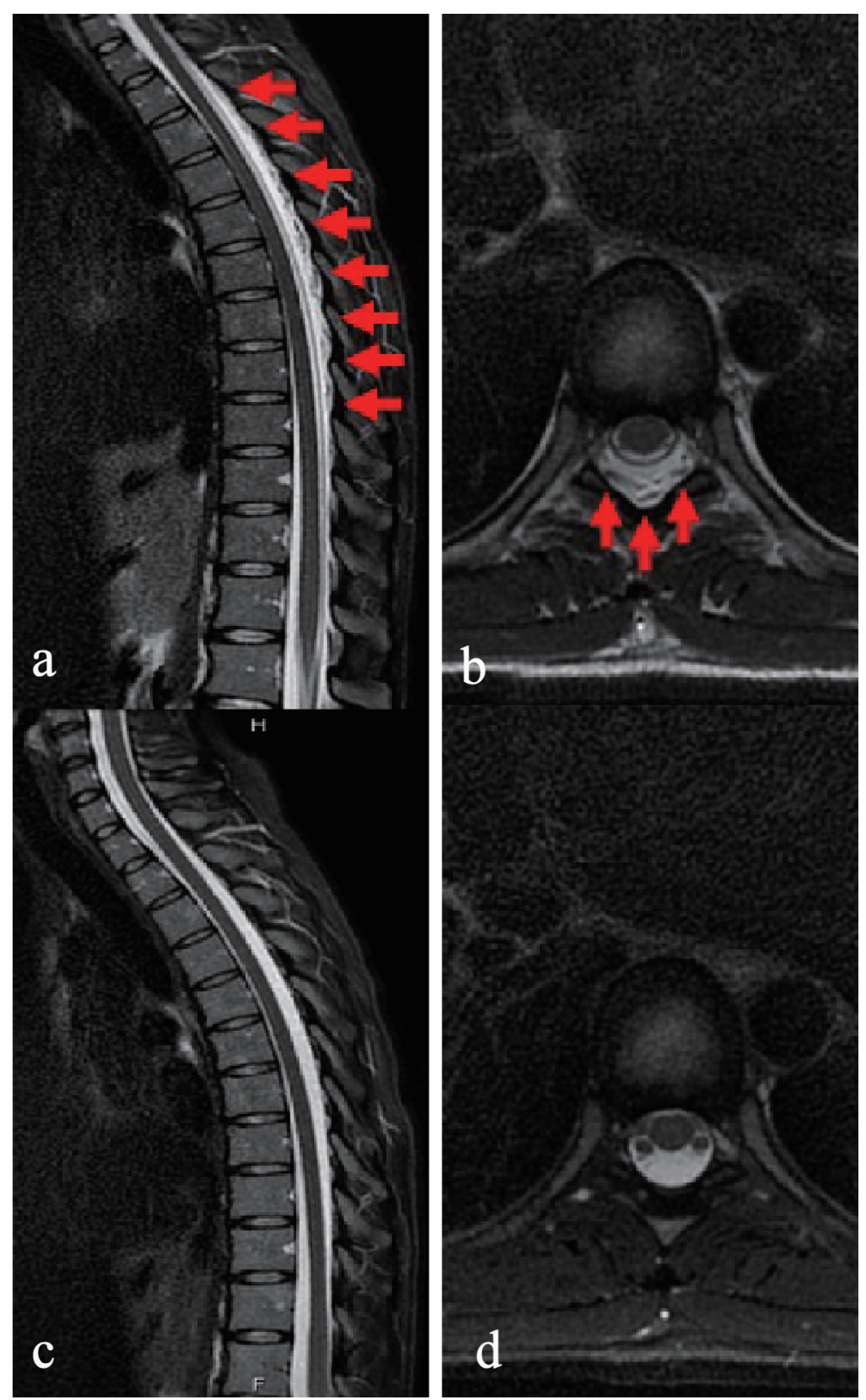

Figure 1. Magnetic resonance imaging T2-weighted image in thoracic vertebrae. (a) Sagittal imaging on admission. A 0.5-mmthick layer of spinal epidural fluid in the posterior epidural space in the whole thoracic region of the spinal cord, confirming a compressed epidural space (red arrow). (b) Axial imaging on admission. Similar findings to (a) are confirmed (red arrow). (c) Sagittal imaging, 30 days into antibiotics treatment. Findings in (a) have disappeared. (d) Axial imaging, 30 days into antibiotics treatment. Thickness of spinal epidural fluid in the posterior epidural space is reduced, and compression of the epidural space has improved.

agnosis can lead to potentially devastating consequences [11]. Therefore, clinicians should investigate whether this infection is present in patients complaining of back pain who have the following risk factors, because they all raise suspicions of SEA: DM (the most common risk factor); immunosuppression; malignancy; cirrhosis; chronic kidney disease; regular alcohol use; HIV infection; rheumatoid arthritis; any bacteremia risk factor (predisposing factors such as intramuscular injections, IV drug use, or indwelling vascular device); or clinical fea- tures such as back pain, often with insidious onset, worsening at night; focal associated with other systemic symptoms (e.g., anorexia, lethargy, weight loss, vomiting, and fever); focal neurologic symptoms; limb weakness; dysesthesias; radicular pain; gait disturbance, and bowel or bladder dysfunction [11, 17]. The current optimal strategy for SEA diagnoses involves a transition from a "classic triad" screening to a risk-factorbased model [7]. In this case, the patient had all of the classic triad symptoms, making it straightforward to consider SEA in 


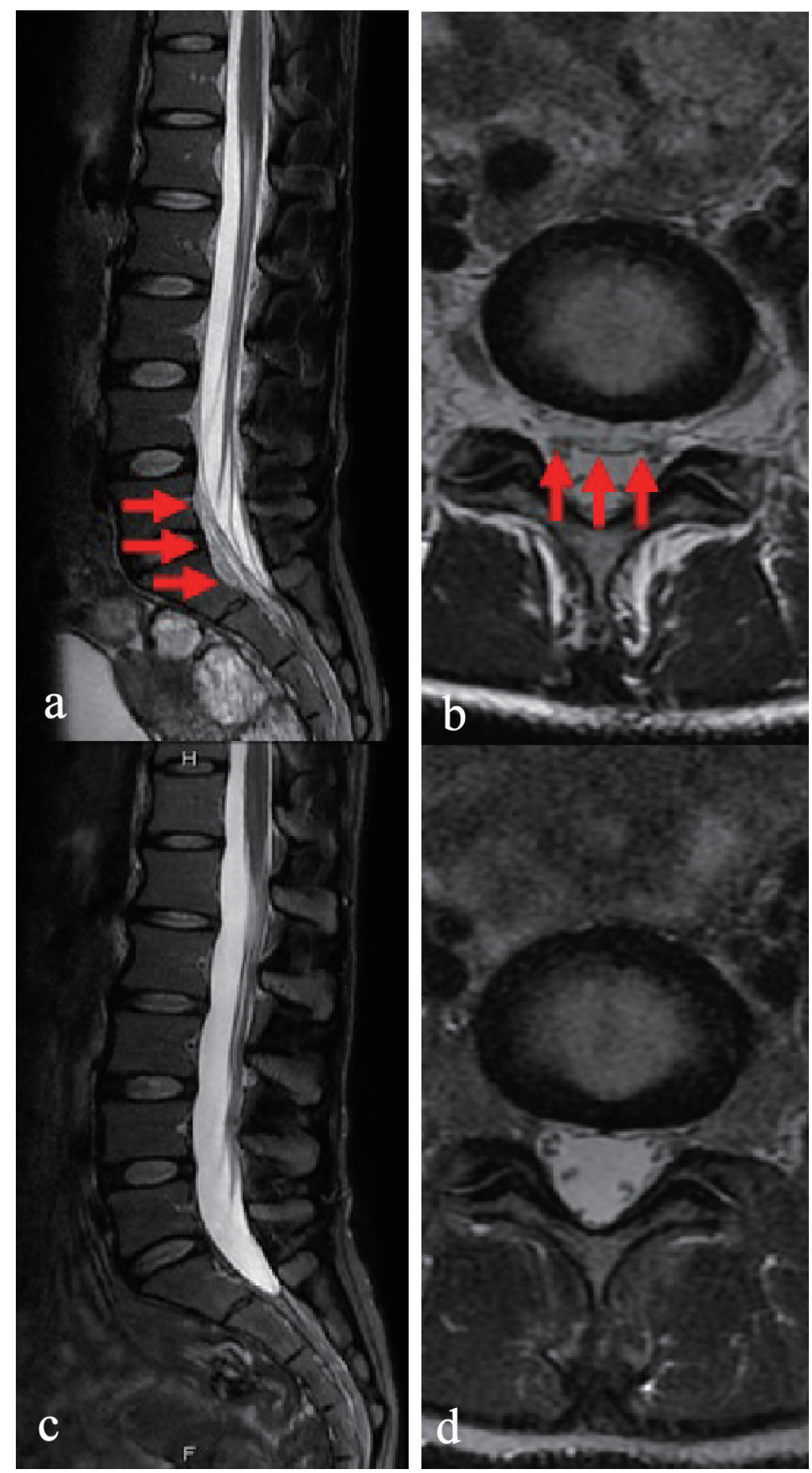

Figure 2. Magnetic resonance imaging T2-weighted image in lumber and sacral vertebrae. (a) Sagittal imaging on admission. A 0.5-mm-thick layer of spinal epidural fluid in the anterior epidural space from the L5 to S2 vertebral body level region, confirming a compressed epidural space (red arrow). (b) Axial imaging on admission. Similar findings to (a) are not confirmed. (c) Sagittal imaging, 30 days into antibiotics treatment. Findings in (a) have disappeared. (d) Axial imaging, 30 days into antibiotics treatment. Findings in (a) are not confirmed.

the differential diagnosis.

Further diagnostic testing using laboratory and imaging evaluation is required when considering SEA in the differential diagnosis $[10,11]$. Common blood tests used for diagnostic evaluation of suspected SEA patients include $\mathrm{CBC}$ with differential, ESR, and CRP [11]. These tests have rapid results, generally available within $1-2 \mathrm{~h}[11]$. Blood cultures can also be drawn upon initial evaluation, though the results are not 
immediately available [11]. Blood tests are not entirely reliable indicators of the presence of disease, but they can be used alongside clinical suspicion, history and physical exam findings, and diagnostic imaging in order to confirm diagnoses [11]. In CBC, leukocytosis presence can vary dramatically (38$80 \%)$ [11]. Moderate WBC elevation $\left.\left(11.0-17.0 \times 10^{3} / \mu \mathrm{L}\right)\right)$ is the most common, though wide ranges have been noted, and there is not necessarily a direct predictive correlation between degree of elevation and disease severity [11]. WBC count is highly nonspecific, and a lack of leukocytosis should not be regarded as excluding an SEA diagnosis [11]. ESR is a significantly more sensitive indicator than leukocytosis for early detection of the disease process, and should be obtained on any patients considered to have a possibility of SEA [11]. Sensitivity ranges from $68-100 \%$; however, in the $68 \%$ sensitivity case series, only an ESR of $>50 \mathrm{~mm} / \mathrm{h}$ was considered positive (0 $-20 \mathrm{~mm} / \mathrm{h}$ is considered the normal range) [11]. When any $>$ $20 \mathrm{~mm} / \mathrm{h}$ value is regarded as abnormal, sensitivity increases to $84-100 \%$ [11]. In a meta-analysis of 915 patients with confirmed cases of SEA, ESR at the initial evaluation was noted to have a sensitivity of $94 \%$ [11]. CRP has a similar sensitivity of $84-100 \%$ in confirmed cases of SEA; because its serum concentrations rise faster than ESR or WBC, and because it is less affected by other plasma factors, it may be a more effective early disease marker [11]. CRP concentrations also rapidly decrease alongside disease resolution, and can be used as a guide of time to treatment completion [11].

Regarding microbial diagnosis, blood cultures and abscess and spine cultures are important. In the event of a positive blood culture, a spinal biopsy is unnecessary, as the bloodstream organism is generally the organism in the SEA [11]. Whenever possible, cultures should be obtained from two separate peripheral venipuncture sites [11]. Blood culture sensitivity varies, with a range of 31-68\% [11]. Positive cultures are more likely in the event of a more severe, disseminated infection, and less likely in patients with localized infections (discitis) [11]. Staphylococcus aureus is the most commonly isolated pathogen, although gram-negative organisms are increasingly prevalently found in IV drug users [11, 18]. Blood culture results take $24-48 \mathrm{~h}$; as a result, they can rarely be used for initial diagnostic or treatment decision guidance [11]. However, blood culture and direct tissue culture results are used together for causative organism confirmation, as well as to help determine the course of antibiotic treatment in the event of a negative biopsy tissue culture [11]. Note as well that urinary tract infections are frequently overlooked as a source of bacteremia; consequently, urine cultures should be ordered on all patients suspected of SEA [11].

Radiological evaluations play an important role in both diagnosis and management of SEA, with modern radiological techniques such as CT and MRI scans providing tremendous aid $[2,10]$. Of these, MRI scans have become the gold standard for diagnosing SEA $[3,11]$. If a patient has findings raising suspicions of SEA, and has only been evaluated with a lumbosacral spine MRI study, consider additional imaging that incorporates the thoracic spine at a minimum [11]. Ideally, complete spinal imaging should be performed as an occult lesion assessment [11]. Additionally, a gadolinium-enhanced MRI scan is considered the modality of choice for spinal infec- tion diagnosis, evaluation, treatment planning, interventional treatment, and treatment monitoring [3, 7, 17].

Additionally, clinicians should order a tuberculosis blood test (tuberculosis-QuantiFERON), or perform a tuberculosis skin test at the time of initial evaluation, for patients with suspected cases of SEA who also have risk factors for active tuberculosis [11]. Compared to many parts of Asia, tuberculous epidural abscess is less prevalent in the USA, but it must still be regarded as a potential etiology in at-risk patients as appropriate [11].

Successful SEA diagnosis and management requires neurologists, neurosurgeons, radiologists, and infectious disease physicians to work together $[5,14]$. At present, medical management and surgical intervention are the two main SEA treatment options [3]. Generally, medical management is reserved for patients whose significant comorbidities contraindicate surgery, patients with significantly extended abscesses, or patients with no neurological deficits or neurological deficits lasting more than $48-72 \mathrm{~h} \mathrm{[3].} \mathrm{A} \mathrm{CT-guided} \mathrm{percutaneous}$ needle aspiration of the abscess may also accompany medical management [3, 19]. However, little consensus exists regarding optimal SEA treatment; furthermore, it is unclear whether medical or surgical treatment would be the ideal initial approach for patients who are neurologically intact $[3,8]$.

In the vast majority of cases, treatment consists of a combination of both antibiotics and surgical intervention [6]. While surgery remains the mainstream treatment, SEA can be managed for a certain subset of patients without operative intervention, and select cases offer the possibility of medical management of SEA, similarly to VO, with biopsy and antibiotics $[11,14]$. The criteria reported for non-operative management include: 1) A known pathogenic organism, neurologic stability, MRI or CT scan availability to enable rapid follow-up, and close serial neurosurgical or nursing monitoring; 2) Patients who have extended-duration pre-existing paralysis $(>36-48$ h) may not need surgical intervention; and 3) Urgent surgical intervention may be precluded by significant medical comorbidities [11]. In the event that patients are hemodynamically unstable, have abnormal neurological findings, or have positive blood cultures, clinicians should immediately start antibiotics [11]. In patients without these findings, do not begin antibiotics until after an image-guided biopsy can be performed [11]. A positive blood culture indicates that spinal biopsy is unnecessary, as the bloodstream organism will usually be the organism in the SEA [11]. The treatment of choice for an extensive SEA will generally be urgent surgical decompression combined with long-term antibiotic treatment [4]. Regarding treatment of SEA with antibiotics, because Staphylococcus aureus is the most common microorganism, it is essential to target staphylococci with the antimicrobial therapy [11]. As of the guideline's writing, Staphylococcus aureus (methicillinresistant Staphylococcus aureus (MRSA)) has approximately $50 \%$ methicillin resistance, and as a result, vancomycin is regarded as the treatment of choice [11]. Treatment doses should be provided that will achieve a $15-20 \mu \mathrm{g} / \mathrm{mL}$ vancomycin trough level [11].

Regarding surgery, minimally invasive surgical techniques have demonstrated great potential to be safe and effective, with low surgical morbidity and rapid patient recovery $[4,20]$. 
When surgical intervention is chosen as the treatment regimen, the primary goals include decompressing the epidural space, draining the abscess, achieving spinal stability, and sampling the abscess to identify the pathogen [3].

As a prognosis, SEA diagnosis and treatment delays directly correspond to worse, often debilitating, permanent neurologic sequela in patients, and sometimes even death outcomes $[2,5,7,10,16,21]$. By the time the more conspicuous and broad-ranging neurological symptoms have developed, they are often irreversible [7]. In fact, final outcomes are correlated with symptom severity and duration before surgery, and sepsis has an unfavorable prognosis as a factor [7, 12]. In summary, the major prognostic factor for favorable SEA outcomes is early diagnosis, yet it has proven difficult to make this diagnosis in an emergency department setting [7]. Recent evidence demonstrates increasing amounts of initial medical management, but in cases where medical management is unsuccessful, followed by surgical treatment, patients may have a worse prognosis than when initially opting for surgical treatment [8]. Following SEA surgery, the 30-day mortality rate was $3.7 \%$ [21]. The majority of mortalities occurring within 30 days of surgery were within 2 weeks [21]. Independent mortality risk factors included advanced age, diabetes, hypertension, respiratory comorbidities, renal comorbidities, metastatic cancer, and thrombocytopenia [21]. Mortality risk ranged from $0.3 \%$ to $37.5 \%$ based on various risk factors, with septic shock, cardiac arrest, and pneumonia being associated with mortality [21].

This case study has a number of limitations. First, this article reviews only a single case report and case series of SEA; therefore, the actual circumstances of SEA may differ from the results of the literature review, due to reporting bias. Second, we should have performed enhanced CT or/and MRI scans for further diagnosis, because they show contrast effect for abscesses. Third, we should have added vancomycin to cover MRSA as an empiric therapy of antibiotics treatment.

\section{Conclusions}

In conclusion, we have presented the first known case of disseminated SEA in an immunocompetent individual. This case highlights the complicated pathology, diagnosis, and treatment of SEA. On the other hand, further investigations are needed to clarify the precise etiology and diagnosis of SEA. In addition, this case suggests the need for a careful and detailed examination when encountering patients presenting with fever, low back pain even in an immunocompetent individual; we should thoroughly investigate, including further image investigations, bacteriological and pathologic examination.

\section{Acknowledgments}

The author would like to thank Dr. Kento Takeshima, from Department of General Medicine, Kanazawa Medical University Himi Municipal Hospital, for her invaluable help with review of the literature.

\section{Financial Disclosure}

None to declare.

\section{Conflict of Interest}

None to declare.

\section{Informed Consent}

Both written and verbal informed consents were obtained from the patient for publication of this case report and any accompanying images.

\section{Author Contributions}

Usuda D collected the case data, prepared the pictures and table, and wrote the manuscript; all authors proofread the pathologic materials; Taki Y, Izumida T, Sangen R, Higashikawa $\mathrm{T}$, Hatano E, Yokoyama M, and Kasamaki Y proofread and revised the manuscript. All authors approved the final version to be published.

\section{Data Availability}

The authors declare that data supporting the findings of this study are available within the article.

\section{References}

1. Akhondi H, Baker MB. Epidural Abscess. Mar 30, 2020. In: StatPearls [Internet]. Treasure Island (FL): StatPearls Publishing; 2020.

2. Ameer MA, Knorr TL, Mesfin FB. Spinal Epidural Abscess. Mar 31, 2020. In: StatPearls [Internet]. Treasure Island (FL): StatPearls Publishing; 2020.

3. Turner A, Zhao L, Gauthier P, Chen S, Roffey DM, Wai EK. Management of cervical spine epidural abscess: a systematic review. Ther Adv Infect Dis. 2019;6:2049936119863940.

4. Proietti L, Ricciardi L, Noia G, Barone G, Valenzi E, Perna A, Giannelli I, et al. Extensive Spinal Epidural Abscesses Resolved with Minimally Invasive Surgery: Two Case Reports and Review of the Recent Literature. Acta Neurochir Suppl. 2019;125:345-353.

5. Chow F. Brain and Spinal Epidural Abscess. Continuum (Minneap Minn). 2018;24(5, Neuroinfectious Disease):1327-1348.

6. Araujo F, Ribeiro C, Silva I, Nero P, Branco JC. Klebsiella pneumoniae Spinal Epidural Abscess treated conservatively: case report and review. Acta Reumatol Port. 2012;37(3):260-263.

7. Alerhand S, Wood S, Long B, Koyfman A. The time- 
sensitive challenge of diagnosing spinal epidural abscess in the emergency department. Intern Emerg Med. 2017;12(8):1179-1183.

8. Stratton A, Gustafson K, Thomas K, James MT. Incidence and risk factors for failed medical management of spinal epidural abscess: a systematic review and meta-analysis. J Neurosurg Spine. 2017;26(1):81-89.

9. Maiese A, Volonnino G, Viola RV, Nelson Cavallari E, Fazio V, Arcangeli M, La Russa R. A rare case of Spinal Epidural Abscess following mesotherapy: a challenging diagnosis and the importance of clinical risk management. Considerations concerning uncommon risk factor for development of Spinal Epidural Abscess and its prevention. Clin Ter. 2020;170(1):e15-e18.

10. Sasani M, Aydin O, Aydin AL, Oktenoglu T, Ozer AF, Ercelen O. Spinal epidural abscess as a result of dissemination from gluteal abscess secondary to intramuscular analgesic injection. Pain Pract. 2009;9(5):399-403.

11. Chenoweth CE, Bassin BS, Mack MR, Oppenlander ME, Patel RD, Quint DJ, Seagull FJ. In: Vertebral Osteomyelitis, Discitis, and Spinal Epidural Abscess in Adults. Ann Arbor (MI). 2018.

12. Villarejo-Galende A, Camacho-Salas A, Penas-Prado M, Juntas-Morales R, Gonzalez-de la Aleja J, Ramos A, Rodriguez-Vallejo A. [Spinal epidural abscess in a patient with septicaemia]. Rev Neurol. 2003;36(12):1152-1155.

13. LaPenna PA, Roos KL. Bacterial infections of the central nervous system. Semin Neurol. 2019;39(3):334-342.

14. Eltorai AEM, Naqvi SS, Seetharam A, Brea BA, Simon C. Recent developments in the treatment of spinal epidural abscesses. Orthop Rev (Pavia). 2017;9(2):7010.

15. Zhang JH, Wang ZL, Wan L. Cervical epidural analgesia complicated by epidural abscess: A case report and literature review. Medicine (Baltimore). 2017;96(40):e7789.

16. Babu JM, Patel SA, Palumbo MA, Daniels AH. Spinal emergencies in primary care practice. Am J Med. 2019;132(3):300-306.

17. Tali ET, Oner AY, Koc AM. Pyogenic spinal infections. Neuroimaging Clin N Am. 2015;25(2):193-208.

18. Low SY, Ong CW, Hsueh PR, Tambyah PA, Yeo TT. Neisseria gonorrhoeae paravertebral abscess. J Neurosurg Spine. 2012;17(1):93-97.

19. Ran B, Chen X, Zhong Q, Fu M, Wei J. CT-guided minimally invasive treatment for an extensive spinal epidural abscess: a case report and literature review. Eur Spine J. 2018;27(Suppl 3):380-385.

20. Verdu-Lopez F, Vanaclocha-Vanaclocha V, Mayorga-Villa JD. Minimally invasive spine surgery in spinal infections. J Neurosurg Sci. 2017;61(3):303-315.

21. Du JY, Schell AJ, Kim CY, Trivedi NN, Ahn UM, Ahn NU. 30-day mortality following surgery for spinal epidural abscess: incidence, risk factors, predictive algorithm, and associated complications. Spine (Phila Pa 1976). 2019;44(8):E500-E509. 